\title{
Parameterization of Quadrilateral Meshes
}

\author{
Li Liu ${ }^{1}$, CaiMing Zhang ${ }^{1,2}$, and Frank Cheng ${ }^{3}$ \\ ${ }^{1}$ School of Computer Science and Technology, Shandong University, Jinan, China \\ ${ }^{2}$ Department of Computer Science and Technology, Shandong Economic University, Jinan, \\ China \\ ${ }^{3}$ Department of Computer Science, College of Engineering, University of Kentucky, \\ America \\ liuli_790209@163.com
}

\begin{abstract}
Low-distortion parameterization of 3D meshes is a fundamental problem in computer graphics. Several widely used approaches have been presented for triangular meshes. But no direct parameterization techniques are available for quadrilateral meshes yet. In this paper, we present a parameterization technique for non-closed quadrilateral meshes based on mesh simplification. The parameterization is done through a simplify-project-embed process, and minimizes both the local and global distortion of the quadrilateral meshes. The new algorithm is very suitable for computer graphics applications that require parameterization with low geometric distortion.
\end{abstract}

Keywords: Parameterization, mesh simplification, Gaussian curvature, optimization.

\section{Introduction}

Parameterization is an important problem in Computer Graphics and has applications in many areas, including texture mapping [1], scattered data and surface fitting [2], multi-resolution modeling [3], remeshing [4], morphing [5], etc. Due to its importance in mesh applications, the subject of mesh parameterization has been well studied. Parameterization of a polygonal mesh in 3D space is the process of constructing a one-to-one mapping between the given mesh and a suitable 2D domain. Two major paradigms used in mesh parameterization are energy functional minimization and the convex combination approach. Maillot proposed a method to minimize the norm of the Green-Lagrange deformation tensor based on elasticity theory [6]. The harmonic embedding used by Eck minimizes the metric dispersion instead of elasticity [3]. Lévy proposed an energy functional minimization method based on orthogonality and homogeneous spacing [7]. Non-deformation criterion is introduced in [8] with extrapolation capabilities. Floater [9] proposed shape-preserving parameterization, where the coefficients are determined by using conformal mapping and barycentric coordinates. The harmonic embedding $[3,10]$ is also a special case of this approach, except that the coefficients may be negative.

However, these techniques are developed mainly for triangular mesh parameterization. Parameterization of quadrilateral meshes, on the other hand, is 
actually a more critical problem because quadrilateral meshes, with their good properties, are preferred in finite element analysis than triangular meshes. Parameterization techniques developed for triangle meshes are not suitable for quadrilateral meshes because of different connectivity structures.

In this paper, we present a parameterization technique for non-closed quadrilateral meshes through a simplify-project-embed process. The algorithm has the following advantages:(1) the method provably produces good parameterization results for any non-closed quadrilateral mesh that can be mapped to the 2D plane; (2) the method minimizes the distortion of both angle and area caused by parameterization; (3) the solution does not place any restrictions on the boundary shape; (4) since the quadrilateral meshes are simplified, the method is fast and efficient.

The remaining part of this paper is organized as follows. The new model and the algorithm are presented in detail in Section 2. Test results of the new algorithm are shown in Section 3. Concluding remarks are given in Section 4.

\section{Parameterization}

Given a non-closed quadrilateral mesh, the parameterization process consists of four steps. The first step is to get a simplified version of the mesh by keeping the boundary and interior vertices with high Gaussian curvature, but deleting interior vertices with low Gaussian curvature. The second step is to map the simplified mesh onto a 2D domain through a global parameterization process. The third step is to embed the deleted interior vertices onto the $2 \mathrm{D}$ domain through a weighted discrete mapping. This mapping preserves angles and areas and, consequently, minimizes angle and area distortion. The last step is to perform an optimization process of the parameterization process to eliminate overlapping. Details of these steps are described in the subsequent sections.

For a given vertex $v$ in a quadrilateral mesh, the one-ring neighbouring vertices of the vertex $v$ are the vertices that share a common face with $v$. A one-ring neighboring vertex of the vertex $v$ is called an immediate neighboring vertex if this vertex shares a common edge with $v$. Otherwise, it is called a diagonally neighboring vertex.

\subsection{Simplification Algorithm}

The computation process, as well as the distortion, may be too large if the entire quadrilateral mesh is projected onto the plane. To speed up the parameterization and minimize the distortion, we simplify the mesh structure by reducing the number of interior vertices but try to retain a good approximation of the original shape and appearance. The discrete curvature is one of the good criteria of simplification while preserving the shape of an original model.

In spite of the extensive use of quadrilateral meshes in geometric modeling and computer graphics, there is no agreement on the most appropriate way to estimate geometric attributes such as curvature on discrete surfaces. By thinking of a 
quadrilateral mesh as a piecewise linear approximation of an unknown smooth surface, we can try to estimate the curvature of a vertex using only the information that is given by the quadrilateral mesh itself, such as the edge and angles. The estimation does not have to be precise. To speed up the computation, we ignore the effect of diagonally neighboring vertices, and use only immediate neighboring vertices to estimate the Gaussian curvature of a vertex, as shown in Fig.1-(a). We define the integral Gaussian curvature $\bar{K}=\bar{K}_{v}$ with respect to the area $S=S_{v}$ attributed to $v$ by

$$
\bar{K}=\int_{s} K=2 \pi-\sum_{i=1}^{n} \theta_{i}
$$

where $\theta_{i}$ is the angle between two successive edges. To derive the curvature from the integral values, we assume the curvature to be uniformly distributed around the vertex and simply normalized by the area

$$
K=\frac{\bar{K}}{S} .
$$

where $S$ is the sum of the areas of adjacent faces around the vertex $v$. Different ways of defining the area $S$ result in different curvature values. We use the Voronoi area, which sums up the areas of vertex $v$ 's local Voronoi cells. To determine the areas of the local Voronoi cells restricted to a triangle, we distinguish obtuse and nonobtuse triangles as shown in Fig. 1. In the latter case they are given by

$$
S_{A}=\frac{1}{8}\left(\left|v_{i} v_{k}\right|^{2} \cot \left(\gamma_{i}\right)+\left|v_{i} v_{j}\right|^{2} \cot \left(\delta_{i}\right)\right)
$$

For obtuse triangles,

$$
S_{B}=\frac{1}{8}\left|v_{i} v_{k}\right|^{2} \tan \left(\gamma_{i}\right), \quad S_{C}=\frac{1}{8}\left|v_{i} v_{j}\right|^{2} \tan \left(\delta_{i}\right), \quad S_{A}=S-S_{B}-S_{C} .
$$

A vertex deletion means the deletion of a vertex with low Gaussian curvature and the incident edges. During the simplification process, we can adjust the tolerance value to control the number of vertices reduced.

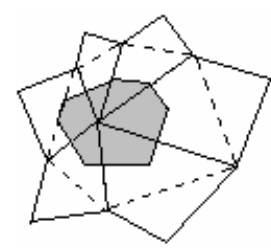

(a)

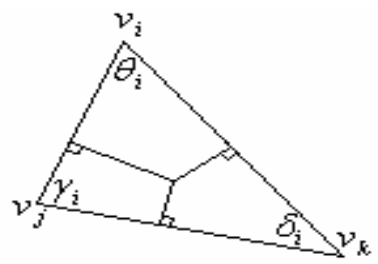

(b)

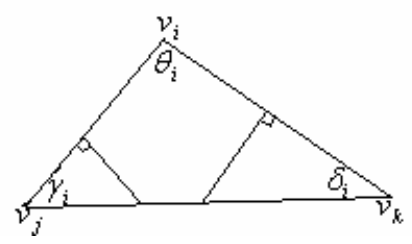

(c)

Fig. 1. Voronoi area. (a) Voronoi cells around a vertex; (b) Non-obtus angle; (c) Obtus angle. 


\subsection{Global Parameterization}

Parameterizing a polygonal mesh amounts to computing a correspondence between the 3D mesh and an isomorphic planar mesh through a piecewise linear mapping. For the simplified mesh $M$ obtained in the first step, the goal here is to construct a mapping between $M$ and an isomorphic planar mesh $U$ in $R^{2}$ that best preserves the intrinsic characteristics of the mesh $M$. We denote by $v_{i}$ the 3D position of the $i$ th vertex in the mesh $M$, and by $u_{i}$ the $2 \mathrm{D}$ position (parameterized value) of the corresponding vertex in the 2D mesh $U$.

The simplified polygonal mesh $M$ approximates the original quadrilateral mesh, but the angles and areas of $M$ are different from the original mesh. We take the edges of the mesh $M$ as springs and project vertices of the mesh onto the parameterization domain by minimizing the following edge-based energy function

$$
\frac{1}{2} \sum_{\{i, j\} \in E d g e} \frac{1}{\left|v_{i}-v_{j}\right|^{r}}\left|u_{i}-u_{j}\right|^{2}, r \geq 0 .
$$

where Edge is the edge set of the simplified mesh. The coefficients can be chosen in different ways by adjusting $r$. This global parameterization process is performed on a simplified mesh (with less vertices), so it is different from the global parameterization and the fixed-boundary parameterization of triangular meshes.

\subsection{Local Parameterization}

After the boundary and interior vertices with high Gaussian curvature are mapped onto a $2 \mathrm{D}$ plane, those vertices with low curvature, are embedded back onto the parametrization plane. This process has great impact on the result of the parametrization. Hence, it should preserve as many of the intrinsic qualities of a mesh as possible. We need to define what it means by intrinsic qualities for a discrete mesh. In the following, the minimal distortion means best preservation of these qualities.

\subsubsection{Discrete Conformal Mapping}

Conformal parameterization preserves angular structure, and is intrinsic to the geometry and stable with respect to small deformations. To flatten a mesh onto a twodimensional plane so that it minimizes the relative distortion of the planar angles with respect to their counterparts in the 3D space, we introduce an angle-based energy function as follows

$$
E_{A}=\sum_{j \in N(i)}\left(\cot \frac{\alpha_{i j}}{4}+\cot \frac{\beta_{i j}}{4}\right)\left|u_{i}-u_{j}\right|^{2} .
$$

where $N(i)$ is the set of immediate one-ring neighbouring vertices, and $\alpha_{i j}, \beta_{i j}$ are the left and opposite angles of $v_{i}$, as shown in Fig. 2-(a). The coefficients in the 
formula (6) are always positive, which reduces the overlapping in the $2 \mathrm{D}$ mesh. To minimize the discrete conformal energy, we get a discrete quadratic energy in the parameterization and it depends only on the angles of the original surface.

\subsubsection{Discrete Authalic Mapping}

Authalic mapping preserves the area as much as possible. A quadrilateral mesh in 3D space usually is not flat, so we cannot get an exact area of each quadrilateral patch. To minimize the area distortion, we divide each quadrilateral patch into four triangular parts and preserve the areas of these triangles respectively. For instance, in Fig. 2-(b) the quadrilateral mesh $v_{i} v_{j} v_{k} v_{j+1}$ is divided into triangular meshes $\Delta v_{i} v_{j} v_{j+1}$, $\Delta v_{i} v_{j} v_{k}, \Delta v_{i} v_{k} v_{j+1}$ and $\Delta v_{j} v_{k} v_{j+1}$ respectively. This changes the problem of quadrilateral area preserving into that of triangular area preserving.

The mapping resulted from the energy minimization process has the property of preserving the area of each vertex's one-ring neighbourhood in the mesh, and can be written as follows

$$
E_{x}=\sum_{j \in N(i)} \frac{\left(\cot \frac{\gamma_{i j}}{2}+\cot \frac{\delta_{i j}}{2}\right)}{\left|v_{i}-v_{j}\right|^{2}}\left|u_{i}-u_{j}\right|^{2} .
$$

where $\gamma_{i j}, \delta_{i j}$ are corresponding angles of the edge $\left(v_{i}, v_{j}\right)$ as shown in Fig. 2-(c). The parameterization deriving from $E_{x}$ is easily obtained, and the way to solve this system is similar to that of the discrete conformal mapping, but the linear coefficients now are functions of local areas of the 3D mesh.

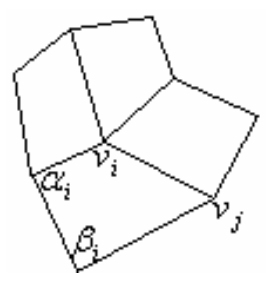

(a)

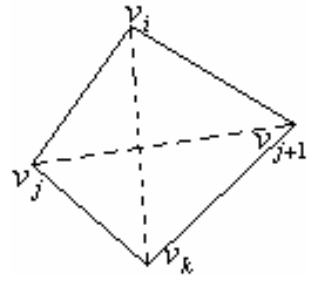

(b)

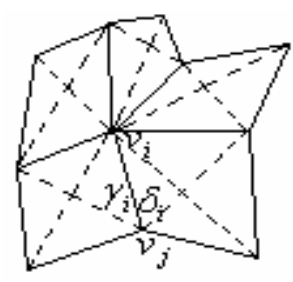

(c)

Fig. 2. Edge and angles. (a) Edge and opposite left angles in the conformal mapping; (b) Quadrilateral mesh divided into four triangles; (c) Edge and angles in the authalic mapping.

\subsubsection{Weighted Discrete Parameterization}

Discrete conformal mapping can be seen as an angle preserving mapping which minimizes the angle distortion for the interior vertices. The resulting mapping will preserve the shape but not the area of the original mesh. Discrete authalic mapping is area preserving which minimizes the area distortion. Although the area of the original 
mesh would locally be preserved, the shape tends to be distorted since the mapping from $3 \mathrm{D}$ to $2 \mathrm{D}$ will in general generate twisted distortion.

To minimize the distortion and get better parameterization results, we define linear combinations of the area and the angle distortions as the distortion measures. It turns out that the family of admissible, simple distortion measures is reduced to linear combinations of the two discrete distortion measures defined above. A general distortion measure can thus always be written as

$$
E=q E_{A}+(1-q) E_{X} .
$$

where $q$ is a real number between 0 and 1. By adjusting the scaling factor $q$, parameterizations appropriate for special applications can be obtained.

\subsection{Mesh Optimization}

The above parameterization process does not impose restriction, such as convexity, on the given quadrilateral mesh. Consequently, overlapping might occur in the projection process. To eliminate overlapping, we optimize the parameterization mesh by adjusting vertex location without changing the topology. Mesh optimization is a local iterative process. Each vertex is optimized for a new location in a number of iterations.

Let $u_{i}^{q}$ be the $q$ times iteration location of the parameterization value $u_{i}$. The optimisation process to find the new location in iterations is the following formula

$$
u_{i}^{q}=u_{i}^{q-1} \lambda_{i} \sum_{i=1}^{n}\left(\frac{u_{j}^{q-1}-u_{i}^{q-1}}{n}\right)+\lambda_{2} \sum_{i=1}^{n}\left(\frac{u_{k}^{q-1}-u_{i}^{q-1}}{n}\right), 0<\lambda_{1}+\lambda_{2}<1 .
$$

where $u_{j}, u_{k}$ are the parameterization values of the immediate and diagonal neighbouring vertices respectively. It is found that vertex optimization in the order of "worst first" is very helpful. We define the priority of the vertex follows

$$
\sigma=\lambda_{i} \sum_{i=1}^{n}\left(\frac{u_{j}^{q-1}-u_{i}^{q-1}}{n}\right)+\lambda_{2} \sum_{i=1}^{n}\left(\frac{u_{k}^{q-1}-u_{i}^{q-1}}{n}\right)
$$

The priority is simply computed based on shape metrics of each parameterization vertex. For a vertex with the worst quality, the highest priority is assigned. Through experiments, we find that more iterations are needed if vertices are not overlapped in an order of "first come first serve". Besides, we must point out that the optimization process is local and we only optimize overlapping vertices and its one-ring vertices, which will minimize the distortion and preserve the parameterization results better.

\section{Examples}

To evaluate the visual quality of a parameterization we use the checkerboard texture shown in Fig. 3, where the effect of the scaling factor $q$ in Eq. (8) can be found. In 
fact, while $q$ is equal to 0 or 1 , the weighted discrete mapping is discrete conformal mapping and authalic mapping separately. We can find few parameterization methods of quadrilateral meshes, so the weighted discrete mapping is compared with discrete conformal mapping and authalic mapping of quadrilateral meshes with $q=0$ and $q=1$ in Eq. (8) separately.

Fig. 3-(a) and (e) show the sampled quadrilateral meshes. Fig. 3-(b) and (f) show the models with a checkerboard texture map using discrete conformal mapping with $q=0$. Fig.3-(c) and (g) show the models with a checkerboard texture map using weighted discrete mapping with $q=0.5$. Fig. 3 -(d) and (h) show the models with a checkerboard texture map using discrete authalic mapping with $q=1$. It is seen that the results using weighted discrete mapping is much better than the ones using discrete conformal mapping and discrete authalic mapping.

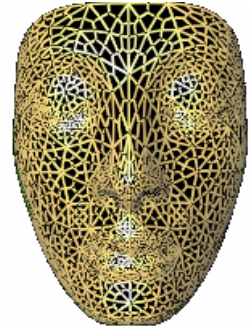

(a)

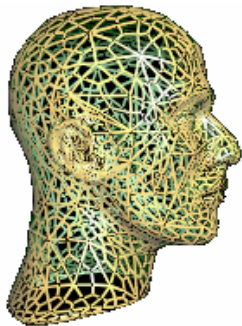

(e)

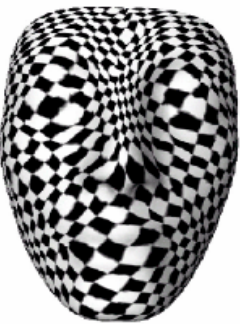

(b)

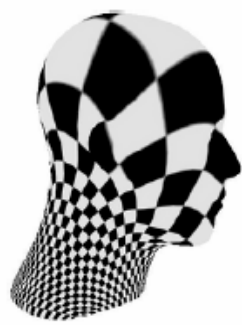

(f)



(c)

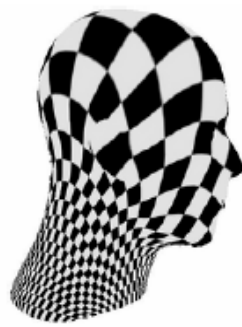

(g)

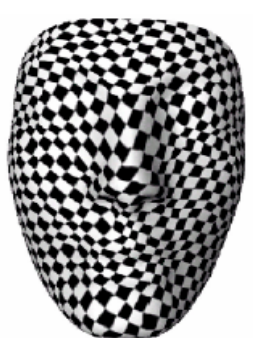

(d)

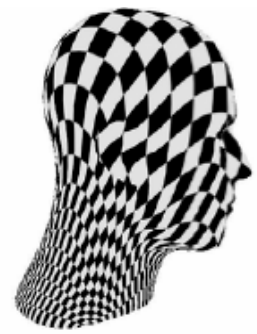

(h)

Fig. 3. Texture mapping. (a) and (e) Models; (b) and (f) Discrete conformal mapping , q=0; (c) and (g) Weighted discrete mapping, $\mathrm{q}=0.5$; (d) and (h) Discrete Authalic mapping, $\mathrm{q}=1$.

The results demonstrate that the medium value (about 0.5) can get smoother parameterization and minimal distortion energy of the parameterization. And the closer $q$ to value 0 or 1 , the larger the angle and area distortions are.

\section{Conclusions}

A parameterization technique for quadrilateral meshes is based on mesh simplification and weighted discrete mapping is presented. Mesh simplification 
reduces computation, and the weighted discrete mapping minimizes angle and area distortion. The scaling factor $q$ of the weighted discrete mapping provides users with the flexibility of getting appropriate parameterisations according to special applications, and establishes different smoothness and distortion.

The major drawback in our current implementation is that the proposed approach may contain concave quadrangles in the planar embedding. It is difficult to make all of the planar quadrilateral meshes convex, even though we change the triangular meshes into quadrilateral meshes by deleting edges. In the future work, we will focus on using a better objective function to obtain better solutions and developing a good solver that can keep the convexity of the planar meshes.

\section{References}

1. Levy, B.: Constrained texture mapping for polygonal meshes. In: Fiume E, (ed.): Proceedings of Computer Graphics. ACM SIGGRAPH, New York (2001) 417-424

2. Alexa, M.: Merging polyhedron shapes with scattered features. The Visual Computer. 16 (2000): 26-37

3. Eck, M., DeRose, T., Duchamp, T., Hoppe, H., Lounsbery, M., Stuetzle, W.: Multiresolution analysis of arbitrary meshes. In: Mair, S.G., Cook, R.(eds.): Proceedings of Computer Graphics. ACM SIGGRAPH, Los Angeles (1995) 173-182

4. Alliez, P., Meyer, M., Desbrun, M.: Interactive geometry remeshing. In: Proceedings of Computer Graphics.ACM SIGGRAPH, San Antonio (2002) 347-354

5. Alexa, M.: Recent advances in mesh morphing. Computer Graphics Forum. 21(2002) $173-196$

6. Maillot, J., Yahia, H., Verroust, A.: Interactive texture mapping. In: Proceedings of Computer Graphics, ACM SIGGRAPH, Anaheim (1993) 27-34

7. Levy, B., Mallet, J.: Non-distorted texture mapping for sheared triangulated meshes. In: Proceedings of Computer Graphics, ACM SIGGRAPH, Orlando (1998) 343-352

8. Jin, M., Wang, Y., Yau, S.T., Gu. X.: Optimal global conformal surface parameterization. In: Proceedings of Visualization, Austin (2004) 267-274

9. Floater, M.S.: Parameterization and smooth approximation of surface triangulations. Computer Aided Geometric Design.14(1997) 231-250

10. Lee, Y., Kim, H.S., Lee, S.: Mesh parameterization with a virtual boundary. Computer \& Graphics. 26 (2006) 677-686 